\title{
Preclinical investigations of a medium-chain triglyceride:fish oil emulsion. I. Effects of bovine milk lipoprotein lipase on lipid composition
}

\author{
YVON A. CARPENTIER, ISABELLE DUPONT, LAURENCE PORTOIS and WILLY J. MALAISSE \\ Laboratory of Experimental Surgery L. Deloyers, Brussels Free University, B-1070 Brussels, Belgium
}

Received April 10, 2006; Accepted June 9, 2006

\begin{abstract}
The bolus intravenous injection of a novel mediumchain triglyceride:fish oil emulsion (MCT:FO, 8:2, w:w) was recently found to increase within $60 \mathrm{~min}$ the leucocyte and platelet phospholipid content of long-chain polyunsaturated $\omega 3$ fatty acids. The present report deals with the effects of bovine milk lipoprotein lipase on the lipid composition of this emulsion. The results are compared to those obtained with either a pure fish oil emulsion or a medium-chain triglyceride: long-chain triglyceride:fish oil emulsion (MLF, $5: 4: 1)$. Emphasis is placed on i) differences in the fate of distinct fatty acids initially present in the triglycerides, diglycerides and phospholipids, ii) the generation of unesterified fatty acids relative to their initial content in each emulsion, and iii) the time course for these various events. The comparison between the three emulsions under consideration also provides information relevant to their respective sensitivity to lipoprotein lipase and suitability in terms of the generation of distinct unesterified fatty acids, including long-chain polyunsaturated $\omega 3$ fatty acids. Furthermore, attention is drawn to the greater efficiency for the hydrolysis of fatty acids from diglycerides as compared to triglycerides and a transient increase in the paired $\mathrm{C} 8: 0 / \mathrm{C} 10: 0$ ratio in the diglycerides generated from the MCT:FO or MLF emulsion. The present study thus affords novel information relevant to the possible use of the MCT:FO emulsion in human subjects.
\end{abstract}

\section{Introduction}

The bolus intravenous injection of a medium-chain triglyceride: fish oil (8:2, w:w) emulsion (MCT:FO) to normal subjects was recently found to cause within $60 \mathrm{~min}$ a marked and sustained increase in the phospholipid content of eicosapentaenoic acid

Correspondence to: Professor Willy J. Malaisse, Laboratory of Experimental Hormonology, Brussels Free University, 808 Route de Lennik, B-1070 Brussels, Belgium

E-mail: malaisse@ulb.ac.be

Key words: bovine milk lipoprotein lipase, medium-chain triglyceride:fish oil emulsion
(C20:5w3) in white blood cells and platelets and docosahexaenoic acid $(\mathrm{C} 22: 6 \omega 3)$ in platelets (1). It was proposed, therefore, as a tool to increase rapidly the content in long-chain polyunsaturated $\omega 3$ fatty acids of cell phospholipids.

The present series of reports deals with several preclinical investigations which are relevant to such a novel approach for the possible prevention of complications, e.g. cardiac arrhythmia, following surgery or other stress situations in predisposed subjects.

This first report concerns the effects of bovine milk lipoprotein lipase on the concentration of various lipids (triglycerides, diglycerides, monoglycerides, phospholipids and unesterified fatty acids) and their relative richness in distinct fatty acids in the MCT:FO emulsion. For the purpose of comparison, parallel experiments were conducted with a pure fish oil emulsion (FO) and a medium-chain triglyceride:longchain triglyceride:fish oil (5:4:1; w:w:w) emulsion (MLF).

\section{Materials and methods}

The three emulsions used in this study contained $(\mathrm{g} / 100 \mathrm{ml})$ triglycerides and diglycerides (20:0), egg phospholipids (1.2) and glycerol (2.5). The glycerides in the FO emulsion were mainly long-chain polyunsaturated $\omega 3$ fatty acid triglycerides and diglycerides (Table I) from fish oil (20.0). In the MCT: FO emulsion, they consisted of a mixture of medium-chain triglycerides (16.0) and fish oil (4.0). In the MLF emulsion, they entailed medium-chain triglycerides (10.0), long-chain triglycerides (8.0) from soybean oil, and fish oil (2.0).

The hydrolysis of the lipids, as catalyzed by bovine milk lipoprotein lipase, was monitored over 240-min incubation under $\mathrm{H}_{2} \mathrm{O}$-saturated air at $37^{\circ} \mathrm{C}$, samples of the incubation medium being removed at $0,30,60,120$ and $240 \mathrm{~min}$ and examined for the concentration and fatty acid composition of triglycerides, diglycerides, monoglycerides and/or phospholipids, and unesterified fatty acids by methods described elsewhere (2).

The experimental procedure included the following steps. Six-well plates $\left(10 \mathrm{~cm}^{2} /\right.$ well; NUNC, Leuven, Belgium) were first exposed for $30 \mathrm{~min}$ at $20^{\circ} \mathrm{C}$ under sterile conditions to a solution of gelatin $(0.2 \%$; w/v; Sigma Aldrich, Bornem, Belgium) at $20^{\circ} \mathrm{C}$ and, after removal of the aqueous phase, dried over the ensuing $120 \mathrm{~min}$. The wells were then successively exposed for $18 \mathrm{~h}$ at $37^{\circ} \mathrm{C}$ to $3.0 \mathrm{ml}$ of a solution 
Table I. Initial fatty acid content (ponderal \%) of triglycerides in distinct emulsions, in the phospholipids of these emulsions and in the diglycerides of the FO emulsion (experiment 1).

Emulsion FO MCT-FO MLT Phospholipids Diglycerides

\begin{tabular}{|c|c|c|c|c|c|}
\hline C6:0 & - & - & - & - & - \\
\hline C8:0 & - & 54.3 & 27.2 & - & - \\
\hline C10:0 & - & 32.8 & 17.1 & - & - \\
\hline C12:0 & - & - & 0.2 & - & - \\
\hline C14:0 & - & - & 0.2 & - & - \\
\hline C16:0 & 4.1 & 1.9 & 6.2 & 36.7 & 2.4 \\
\hline 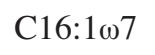 & 0.8 & - & 0.3 & 1.1 & 1.1 \\
\hline C18:0 & 3.1 & - & 2.2 & 23.4 & 3.4 \\
\hline 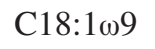 & 6.2 & 2.0 & 10.4 & 17.2 & 4.2 \\
\hline 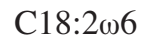 & 3.0 & 1.3 & 25.8 & 11.3 & - \\
\hline C18:3w6 & 1.1 & - & 0.3 & - & 1.2 \\
\hline C20:0 & - & - & - & - & - \\
\hline $\mathrm{C} 18: 3 \omega 3$ & - & - & 4.3 & - & 4.0 \\
\hline C20:1w9 & 2.2 & - & 0.2 & - & - \\
\hline 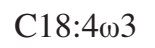 & 2.0 & - & - & - & 2.1 \\
\hline 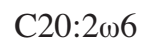 & - & - & - & - & - \\
\hline C20:3w6 & 1.1 & - & - & 1.5 & - \\
\hline C22:0 & 1.7 & - & 0.2 & - & - \\
\hline C20:4w6 & 3.8 & - & 0.2 & 7.6 & 2.3 \\
\hline 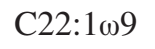 & - & - & - & - & - \\
\hline C20:5w3 & 32.7 & 4.1 & 2.4 & - & 36.5 \\
\hline C24:0 & - & - & - & - & - \\
\hline $\mathrm{C} 22: 3 \omega 3$ & 2.0 & - & - & 1.1 & 2.3 \\
\hline 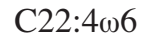 & 1.0 & - & - & - & - \\
\hline C22:5w3 & 4.7 & - & 0.4 & - & 5.2 \\
\hline C22:6w3 & 30.7 & 3.6 & 2.5 & - & 35.4 \\
\hline C26:0 & - & - & - & - & - \\
\hline
\end{tabular}

of heparin sulphate proteoglycan ( $\sim 5 \mu \mathrm{g} / \mathrm{ml}$; Sigma Aldrich) in phosphate-buffered saline (PBS; In Vitrogen, Brussels, Belgium) washed thrice with $\mathrm{Mg}^{2+}$ and $\mathrm{Ca}^{2+}$-free $\mathrm{PBS}$, exposed for $60 \mathrm{~min}$ at $37^{\circ} \mathrm{C}$ to $3.0 \mathrm{ml}$ of a solution of bovine serum albumin (BSA; $1.0 \%$; w/v; In Vitrogen) in PBS, exposed for $30 \mathrm{~min}$ at $20^{\circ} \mathrm{C}$ to $3.0 \mathrm{ml}$ of a solution of bovine milk lipoprotein lipase $(8 \mu \mathrm{g} / \mathrm{ml}$; Sigma Aldrich) in DMEM (In Vitrogen), washed with $3 \mathrm{ml}$ of a solution of BSA (1.0\%; $\mathrm{w} / \mathrm{v}$ ) in DMEM, and eventually washed twice with $3 \mathrm{ml}$ of DMEM.

In order to supply apoproteins to the lipid emulsion, an aliquot $(250 \mu \mathrm{l})$ of each emulsion was incubated for $45 \mathrm{~min}$ at $37^{\circ} \mathrm{C}$ with $5.0 \mathrm{ml}$ of human plasma. After addition of $5.0 \mathrm{ml}$ saline and centrifugation, the lipid emulsion was separated, resuspended in saline and again centrifuged on two successive occasions, before being eventually resuspended in PBS.

Three $\mathrm{ml}$ of each emulsion containing penicillin (50 units/ $\mathrm{ml}$; In Vitrogen), streptomycin (50 $\mu \mathrm{g} / \mathrm{ml}$; In Vitrogen), fetal bovine serum (10\%, v/v; In Vitrogen) and BSA (30 mg/ml; Sigma-Aldrich) were placed in each well. At each sampling time, $0.5 \mathrm{ml}$ was removed for the characterization of glycerides and phospholipids and $0.2 \mathrm{ml}$ for the measurement of unesterified fatty acids.

All results are presented as mean values $( \pm$ SEM or range of individual variation when $n=2$ ) together with the number of individual determinations (n). The statistical significance of differences between mean values was assessed by use of Student's t-test. A geometric mean was used in the analysis of the data mentioned as phospholipid/total loss (\%) in Table II, because these ratios displayed a wide scatter of individual values.

\section{Results}

Two sets of experiments (Expt. 1 and 2) were conducted in which the initial total amount of triglycerides, diglycerides and monoglycerides as judged from the measurements of fatty acids and as expressed in terms of fatty acid content, amounted to $507.9 \pm 10.5 \mu \mathrm{g} / \mathrm{ml}(\mathrm{n}=3)$ in the first series and to $127.2 \pm 0.6 \mu \mathrm{g} / \mathrm{ml}(\mathrm{n}=3)$ in the second series. The lower concentration of lipids in the second set of experiments allowed, at each time point, a more extensive hydrolysis of triglycerides and, hence, a better characterization of the hydrolytic products, especially in the case of the FO emulsion. Moreover, in the second set of experiments, triplicate determinations were performed at each time point in order to assess more precisely the values for each variable under consideration. The results of these two sets of experiments are presented in sequence.

\section{Experiment 1}

Triglycerides. Table I provides the initial fatty acid content (expressed as a ponderal percentage) of triglycerides in the three emulsions (Expt 1). At the onset of incubation, the concentration of triglycerides in the $\mathrm{FO}$ emulsion accounted for only $64.3 \pm 1.8 \%(n=3)$ of the total amount of glycerides, as distinct from $92.6 \pm 1.0 \%(n=6)$ in the two other emulsions.

Relative to the time-zero value, the concentration of triglycerides decreased more rapidly in the case of the MLF and MCT:FO emulsions than in the case of the FO emulsion (Fig. 1, upper panel). As judged from a semi-logarithmic presentation, the decrease in the concentration of triglycerides during incubation failed to display an exponential pattern in the case of the MLF and MCT:FO emulsions.

With the FO emulsion, the relative contribution (ponderal percentage) of $\mathrm{C} 20: 5 \omega 3, \mathrm{C} 22: 5 \omega 3$ and $\mathrm{C} 22: 6 \omega 3$ to the total mass of fatty acids present in the triglycerides slowly and slightly increased during incubation from an initial value of $68.1 \%$ to $72.1 \%$ at $240 \mathrm{~min}$ (Fig. 1, lower panel). This contrasted with the progressively decreasing relative contribution of most other triglyceride-associated fatty acids. For instance, the relative contribution of the various $\mathrm{C} 16$ and $\mathrm{C} 18$ fatty acids fell from an initial value of $20.3 \%$ to a final reading of $17.2 \%$. In the case of either the MLF or MCT:FO emulsion, the quantification of the different fatty acids remaining in triglycerides indicated that the C8:0 and C10:0 fatty acids were the most sensitive to lipolysis. They were indeed the sole fatty acids of which the relative contribution decreased during incubation. For instance, the relative abundance of these two fatty acids represented, at $240 \mathrm{~min}$, only $21.1 \pm 2.0 \%$ 
Table II. Loss of C16:0 and C18:0 from phospholipids and glycerides.

\begin{tabular}{lrrrrrrrr}
\hline \multirow{2}{*}{$\begin{array}{l}\text { Emulsion } \\
\text { Fatty acid }\end{array}$} & \multicolumn{2}{c}{ FO } & & \multicolumn{2}{c}{ MCT:FO } & & \multicolumn{2}{c}{ MLF } \\
\cline { 2 - 3 } & C16:0 & C18:0 & & C16:0 & C18:0 & & C16:0 & C18:0 \\
\hline Loss from phospholipids $(\mu \mathrm{g} / \mathrm{ml})$ & 2.165 & 1.223 & & 2.649 & 1.342 & & 3.201 & 1.460 \\
Loss from diglycerides $(\mu \mathrm{g} / \mathrm{ml})$ & 3.874 & 5.541 & & - & - & & 1.849 & 1.108 \\
Loss from triglycerides $(\mu \mathrm{g} / \mathrm{ml})$ & 5.218 & 3.933 & & 5.678 & - & & 23.497 & 7.801 \\
Total loss $(\mu \mathrm{g} / \mathrm{ml})$ & 11.257 & 10.697 & & 8.327 & 1.342 & & 28.547 & 10.369 \\
Phospholipid/total loss $(\%)$ & 19.230 & 11.430 & & 31.810 & 100.000 & & 11.210 & 14.080 \\
Initial content of diglycerides $(\mu \mathrm{g} / \mathrm{ml})$ & 3.874 & 5.541 & & - & - & & 1.849 & 1.108 \\
Initial content of triglycerides $(\mu \mathrm{g} / \mathrm{ml})$ & 14.403 & 11.032 & & 9.249 & - & & 29.003 & 10.371 \\
Initial content of glycerides $(\mu \mathrm{g} / \mathrm{ml})$ & 18.277 & 16.573 & & 9.249 & - & & 30.852 & 11.479 \\
\hline
\end{tabular}
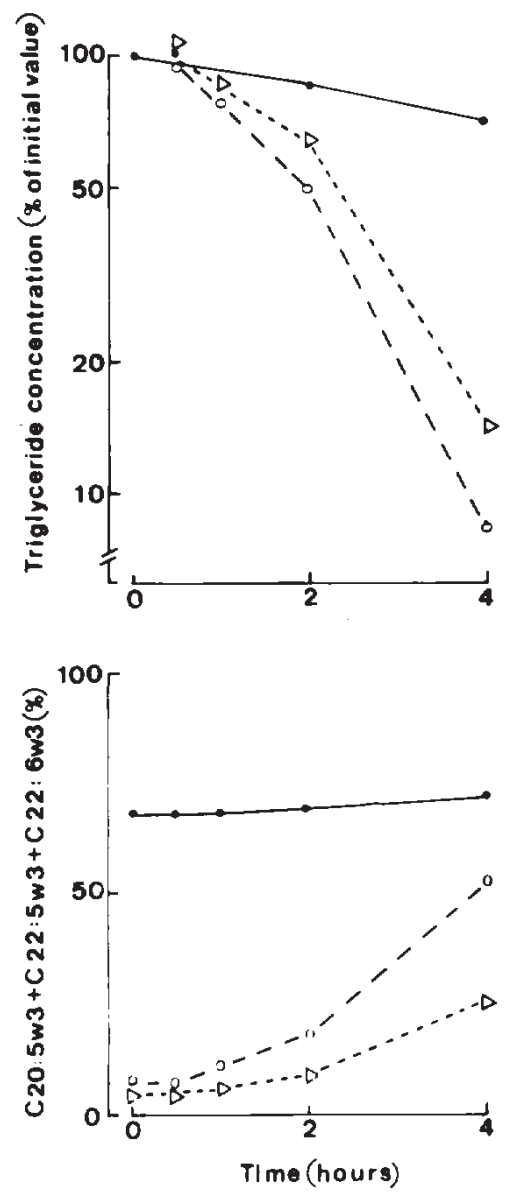

Figure 1. Time course for the changes in triglyceride concentration and composition in the FO (closed circles and solid line), MCT:FO (open circles and dashed line) and MLF (triangles and dotted line) emulsions (Expt. 1). Upper panel: the triglyceride concentration is expressed, in each case, relative to its initial value $(0 \mathrm{~h})$ and ranged on a logarithmic scale. Lower panel: relative contribution of $\mathrm{C} 20: 5 \omega 3, \mathrm{C} 22: 5 \omega 3$ and $\mathrm{C} 22: 6 \omega 3$ to the total amount of fatty acids in the triglyceride fraction.

$(n=4)$ of their paired initial value. In mirror image, the relative contribution of $\mathrm{C} 20: 5 \omega 3$ and $\mathrm{C} 22: 6 \omega 3$ progressively increased in the triglycerides of the MLF and MCT:FO emulsions (Fig. 1, lower panel). The paired $\mathrm{C} 8: 0 / \mathrm{C} 10: 0$ ratio in the triglycerides of the MLF and MCT:FO emulsions decreased from a mean
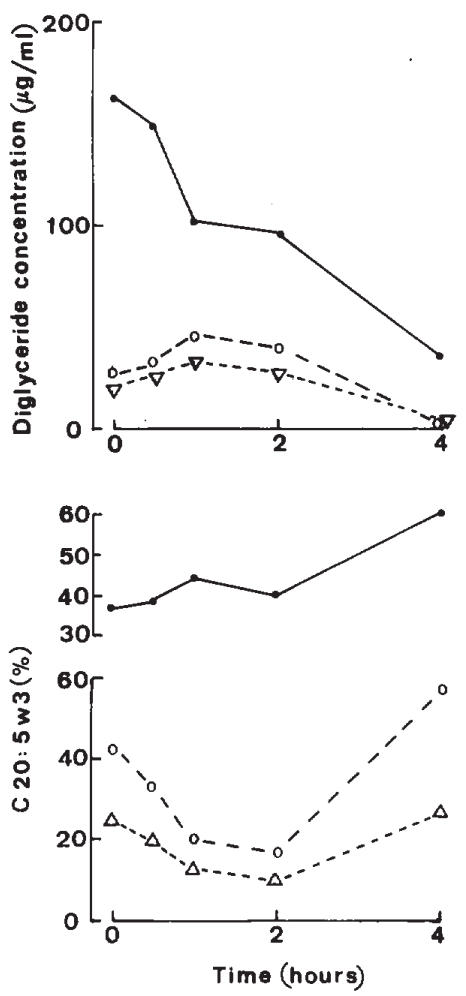

Figure 2. Time course for the changes in diglyceride concentration and composition in the FO (closed circles and solid line), MCT:FO (open circles and dashed line) and MLF (triangles and dotted line) emulsions (Expt. 1). Upper panel: the absolute concentration of diglycerides is ranged on a cartesian scale. Lower panel: relative contribution of C20:5 33 to the total amount of fatty acids in the diglyceride fraction.

value of $1.63 \pm 0.02(\mathrm{n}=4)$ at $0-30 \mathrm{~min}$ to $1.58 \pm 0.01,1.41 \pm 0.01$ and $1.24 \pm 0.09(\mathrm{n}=2$ in each case) at 60,120 and $240 \mathrm{~min}$, respectively, indicating a greater lability of the ester bound involving $\mathrm{C} 8: 0$ as compared to $\mathrm{C} 10: 0$.

Diglycerides. The hydrolysis of triglycerides coincided with the appearance of non-esterified fatty acids, monoglycerides and, on occasion, diglycerides in the incubation medium.

With the FO emulsion, the concentration of diglycerides was highest at the onset of incubation (Fig. 2, upper panel). At that time, it represented, expressed as their fatty acid 
content, $31.3 \%$ of the total amount of glycerides, as distinct from only $4.7 \pm 0.7 \%$ in the case of the MLF and MCT:FO emulsions. Thus, the amount of diglycerides initially present in the emulsions was grossly proportional to their relative content in fish oil.

Fig. 2 (upper panel) documents that the amount of diglycerides present in the FO emulsion progressively decreased during incubation, whilst a transient increase in diglyceride concentration was observed with the MCT:FO and MLF emulsions. In the latter two cases, the peak value was recorded after 60 -min incubation. It averaged $163.7 \pm 1.3 \%(n=2)$ of the paired initial measurement.

The fatty acid composition of these diglycerides was also somewhat different with the three emulsions. With the FO emulsion, the relative abundance of $\mathrm{C} 20: 5 \omega 3, \mathrm{C} 22: 5 \omega 3$ and $\mathrm{C} 22: 6 \omega 3$ averaged at the early times of incubation (0-30 $\mathrm{min})$, respectively, $37.5 \pm 1.0 \%, 5.3 \pm 0.1 \%$ and $36.1 \pm 0.7 \%(\mathrm{n}=2$ in all cases), the $\mathrm{C} 20: 5 \omega 3$ and $\mathrm{C} 22: 6 \omega 3$ fatty acids being the sole ones eventually remaining in detectable amounts in the diglycerides after $4 \mathrm{~h}$ of incubation. In the MLF and MCT:FO emulsions, the $\mathrm{C} 20: 5 \omega 3$ and $\mathrm{C} 22: 6 \omega 3$ were also the predominant fatty acids present in the diglycerides at the onset of incubation, representing 24.2-42.4 and 28.0-39.9\% respectively in these two emulsions. This reinforces the view that the small amount of diglycerides initially present in the latter two emulsions corresponded mainly, if not exclusively, to the contribution of fish oil. Thereafter, the relative amount of C20:5 33 first decreased and then increased (Fig. 2, lower panel), whilst that of $\mathrm{C} 22: 6 \omega 3$ displayed in both the MLF and MCT:FO emulsions a progressive fall.

The different time-related patterns for the fractional abundance of long-chain polyunsaturated $\omega 3$ fatty acids in the diglycerides, as documented in the case of $\mathrm{C} 20: 5 \omega 3$ (Fig. 2, lower panel), most likely reflects the transient accumulation of diglycerides derived from the medium-chain fatty acid triglycerides in the MCT:FO and MLF emulsions. This view is supported by the finding that, with the latter two emulsions, the absolute values $(\mu \mathrm{g} / \mathrm{ml})$ for C8:0 in the diglycerides increased over the first $60 \mathrm{~min}$ of incubation at a mean rate of $11.08 \pm 1.73 / \mathrm{h}(\mathrm{n}=4)$, and then declined to reach a level slightly lower than the paired time-zero measurement. Likewise, with the MCT:FO and MLF emulsions, the absolute values for C10:0 in the diglycerides increased over the first $60 \mathrm{~min}$ of incubation at a mean rate of $5.00 \pm 0.83 / \mathrm{h}(\mathrm{n}=4)$, remained at a fairly stable level during the second hour of incubation and then again decreased to reach values somewhat lower than the initial reading (time-zero) at $240 \mathrm{~min}$. In sharp contrast to these findings, the absolute values for all other fatty acids in the diglycerides of the MCT:FO and MLF emulsions

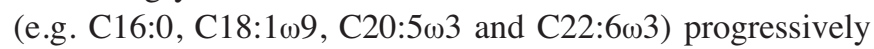
decreased from the 60th min of incubation onwards.

In this first set of experiments, a modest but sizeable contribution of C8:0 and C10:0 to the fatty acids of diglycerides was detected at the onset of incubation in the MCT:FO and MLF emulsions. At that time, the relative contribution of these two fatty acids amounted to 17.7 and $13.7 \%$ with the MCT:FO and MLF emulsions, respectively, with a mean C8:0/C10:0 paired ratio of $1.35 \pm 0.01(n=2)$. Unexpectedly, such a paired ratio first increased $(\mathrm{P}<0.005)$ to $1.88 \pm 0.02$ and $2.01 \pm 0.02$ at 30 and $60 \mathrm{~min}$, respectively, and then fell to
$1.44 \pm 0.06$ at $120 \mathrm{~min}$ and $1.23 \pm 0.04$ at $240 \mathrm{~min}(\mathrm{n}=2$ in all cases). This indicates that the diglycerides generated from the triglycerides present in the MCT:FO and MLF emulsions unexpectedly displayed a high $\mathrm{C} 8: 0 / \mathrm{C} 10: 0$ ratio, largely in excess of that found at the onset of incubation in either the diglycerides $(1.35 \pm 0.01 ; \mathrm{n}=2)$ or triglycerides $(1.63 \pm 0.03 ; \mathrm{n}=2)$. The later fall in the $\mathrm{C} 8: 0 / \mathrm{C} 10: 0$ ratio of diglycerides, which paralleled the fall in the concentration of such diglycerides (Fig. 2, upper panel), could again reflect the greater lability of the ester bound involving C8:0 as compared to C10:0.

The fractional release of fatty acids was obviously higher from diglycerides than from triglycerides. For instance, with the FO emulsion and after $2 \mathrm{~h}$ of incubation, the residual concentration $(\mu \mathrm{g} / \mathrm{ml})$ of $\mathrm{C} 16: 0$ and $\mathrm{C} 18: 0$, on one hand, and

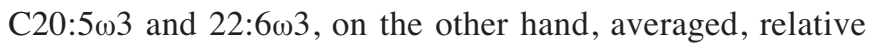
to the corresponding initial value (time-zero), 56.6 \pm 1.9 and $61.6 \pm 3.3 \%$ in the diglyceride fraction, as distinct $(\mathrm{P}<0.02$ or less) from $84.7 \pm 0.0$ and $88.5 \pm 0.1 \%$ in the triglyceride fraction $(n=2$ in all cases).

Monoglycerides. Relatively modest amounts of monoglycerides were also found in the incubation medium. In the case of the FO emulsion, their accumulation was grossly proportional to the length of incubation, with a mean generation rate of $14.1 \pm 1.8 \mu \mathrm{g}$ of fatty acid equivalent per hour $(\mathrm{n}=4)$. In the case of the MCT:FO and MLF emulsions, however, the accumulation of monoglycerides displayed a sigmoidal timerelated pattern. Thus, relative to the peak value reached both with the MCT:FO emulsion $(95.4 \mu \mathrm{g} / \mathrm{ml})$ and MLF emulsion $(72.5 \mu \mathrm{g} / \mathrm{ml})$ after $2 \mathrm{~h}$ of incubation, the readings recorded at 30,60 and $240 \mathrm{~min}$, respectively, averaged $7.4 \pm 0.6 \%$, $29.6 \pm 2.6 \%$ and $87.2 \pm 9.6 \%$ ( $n=2$ in all cases).

In the case of the FO emulsion, the fatty acids present in these monoglycerides were, between 60 and $240 \mathrm{~min}$, mainly

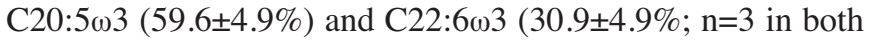
cases). In the case of the MCT:FO emulsion, the fatty acids of monoglycerides consisted, over the same period, mainly of C8:0 $(47.3 \pm 2.7 \%)$ and C10:0 (36.9 $\pm 1.5 \%)$, with a minor contribution of $\mathrm{C} 20: 5 \omega 3(3.9 \pm 1.0 \%)$. Last, in the case of the MLF emulsion, the predominant monoglyceride fatty acids were, between 60 and $240 \mathrm{~min}, \mathrm{C} 8: 0(36.7 \pm 1.1 \%), \mathrm{C} 10: 0$ $(36.9 \pm 1.1 \%)$ and $C 18: 2 \omega 6(19.4 \pm 3.3 \%)$, with again only a minor contribution of $\mathrm{C} 20: 5 \omega 3(3.7 \pm 2.3 \%$; $\mathrm{n}=3$ in all cases $)$. The relative contribution of distinct fatty acids in the monoglycerides was thus grossly comparable, albeit not identical, to that of the same fatty acids in the initial triglyceride fraction.

At variance with the initial situation in the triglyceride fraction (Table I), the amount of C20:5 03 found in the monoglycerides always largely exceeded that of $\mathrm{C} 22: 6 \omega 3$. With the FO emulsion, the $\mathrm{C} 20: 5 \omega 3 / \mathrm{C} 22: 6 \omega 3$ paired ratio increased from $1.47 \pm 0.07(\mathrm{n}=2)$ at $30-60 \mathrm{~min}$ to 1.77 and 3.07 at 120 and $240 \mathrm{~min}$, respectively. With the MCT:FO and MLF emulsions, C20:5 23 was often the sole long-chain polyunsaturated $\omega 3$ fatty acid identified in the monoglycerides. At $240 \mathrm{~min}$, the amount of C20:5 33 found in the monoglycerides of the FO emulsion $(36.8 \mu \mathrm{g} / \mathrm{ml}), \mathrm{MCT}: \mathrm{FO}$ emulsion $(9.8 \mu \mathrm{g} / \mathrm{ml})$ and MLF emulsion $(4.4 \mu \mathrm{g} / \mathrm{ml})$ was virtually proportional to the initial amount of fish oil in these three emulsions.

The paired C8:0/C10:0 ratio in the monoglycerides of the MCT:FO and MLF emulsions remained fairly stable between 

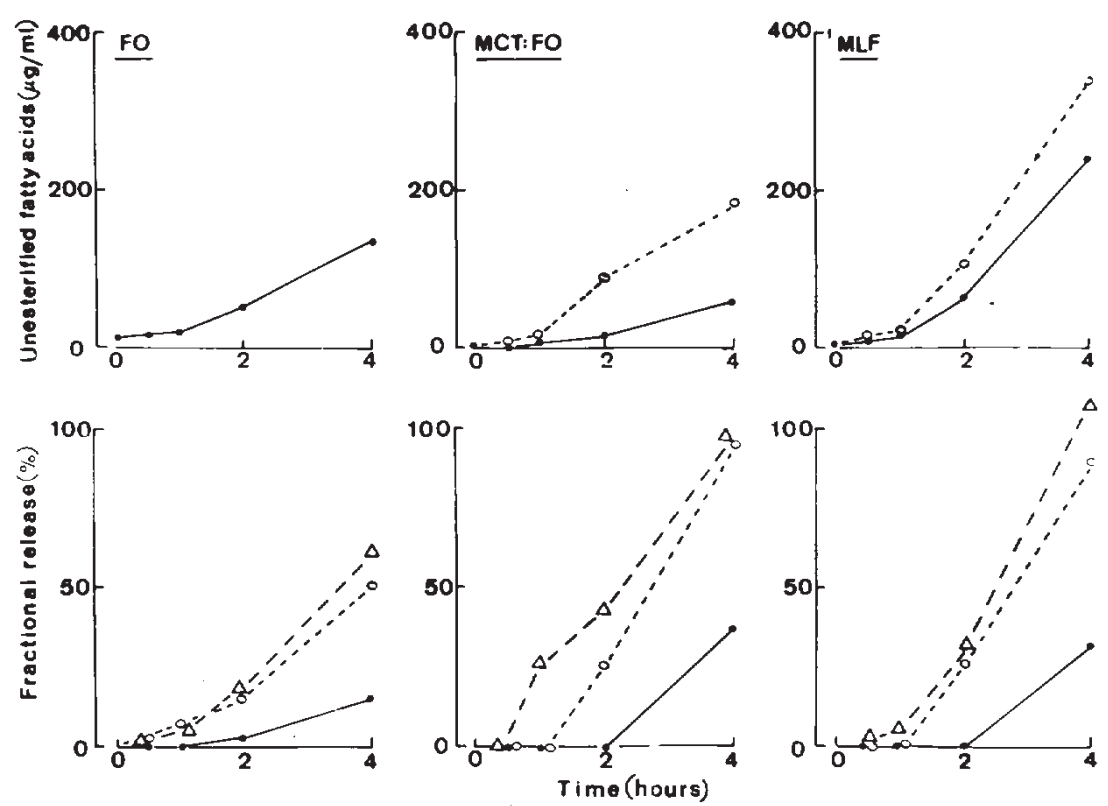

Figure 3. Time course for the changes in unesterified fatty acid concentration and fractional release in the FO (left), MCT:FO (middle) and MLF (right) emulsions (Expt. 1). Upper panel: the total concentrations of fatty acids refer to the measurements made inclusive (open circles and dashed line) or not (closed circles and solid line) of C8:0 and C10:0. Lower panels: fractional release of C16:0 (triangles and dashed line), C22:6w3 (open circles and dotted line) and $\mathrm{C} 20: 5 \omega 3$ (closed circles and solid line), as judged from the ratio between the concentration $(\mu \mathrm{g} / \mathrm{ml})$ of the unesterified fatty acid, at each time point, and its initial concentration in the triglyceride fraction.

30 and $240 \mathrm{~min}$, and was not significantly different between these two emulsions $(\mathrm{P}>0.1)$, with an overall mean value of $1.22 \pm 0.05(\mathrm{n}=8)$. As expected from the greater lability of the ester bound involving C8:0, as compared to C10:0, the latter mean ratio was significantly lower $(\mathrm{P}<0.01)$ than that initially found in the triglyceride fraction $(1.63 \pm 0.03 ; n=2)$.

Unesterified fatty acids. In this first set of experiments, the time course for the accumulation of unesterified fatty acids in the incubation medium displayed, as a rule, an exponential time course (Fig. 3, upper panel). Thus, as judged from the paired difference in concentration of unesterified fatty acids measured at each sampling time, their production rate over the first $60 \mathrm{~min}$ of incubation represented no more than $24.4 \pm 6.1 \%(\mathrm{n}=6 ; \mathrm{P}<0001)$ of the mean corresponding values found thereafter $(100.0 \pm 8.5 \% ; \mathrm{n}=6)$.

It should be noted, however, that the generation of C8:0 and C10:0 could not be assessed in a reliable manner. Indeed the total amount $(\mu \mathrm{g} / \mathrm{ml})$ of these fatty acids recovered as unesterified molecules and in the triglyceride fraction was much lower, after $240 \mathrm{~min}$ of incubation, than that initially present in the triglycerides. As judged from such a comparison, the recovery of these volatile fatty acids was quite variable. It ranged between the extreme values of $21.6 \%$ (C8:0 in the MCT:FO emulsion) and 64.3\% (C10:0 in the MLF emulsion), with in-between values of $41.5 \%$ (C8:0 in the MLF emulsion) and $45.7 \%$ (C10:0 in the MCT:FO emulsion). These percentages indicate that the recovery of C8:0 was always lower than that found, with the same emulsion, in the case of C10:0, as expected from the greater volatility of the former in comparison to the latter fatty acid. They also indicate that, for a given fatty acid, the recovery was lower with the MCT:FO emulsion than with the MLF emulsion. The latter difference can be accounted for by the fact that the initial amount of
C8:0 and $\mathrm{C} 10: 0$ in the triglyceride fraction $(\mu \mathrm{g} / \mathrm{ml})$ was twice higher in the MCT:FO emulsion than in the MLF emulsion (Table I). There was indeed a significant inverse correlation $(\mathrm{r}=-0.967 ; \mathrm{n}=4 ; \mathrm{P}<0.05)$ between the percent recovery (logarithmic values) and the initial corresponding amount $(\mu \mathrm{g} / \mathrm{ml})$ of each fatty acid in the triglyceride fraction.

In the case of other fatty acids, such as C16:0, C20:5 13 and $\mathrm{C} 22: 6 \omega 3$, the total amount found in the various lipid fractions (triglycerides, diglycerides, monoglycerides, phospholipids and unesterified fatty acids) of each emulsion was, whatever the sampling time $(30,60,120$ and $240 \mathrm{~min} ; \mathrm{n}=9$ in each case), never significantly different from the corresponding initial measurement(s), with an overall mean of $99.0 \pm 1.4 \%$ $(n=36)$ relative to the paired reference value at the onset of incubation (time 0).

Relative to the initial amount $(\mu \mathrm{g} / \mathrm{ml})$ of each fatty acid in the triglycerides, the fractional release of $\mathrm{C} 20: 503$ at $240 \mathrm{~min}$ only represented $35.1 \pm 2.4 \%(n=3)$ of that found, with the same emulsion, in the case of $\mathrm{C} 22: 6 \omega 3$; such a percentage being derived from the results obtained with all three emulsions. This indicates a greater lability of ester bound involving C22:6 133 , as compared to $\mathrm{C} 20: 5 \omega 3$. In the case of the FO emulsion, the total amount of long-chain polyunsaturated $\omega 3$ fatty acids found in the incubation medium (i.e. C20:5 13 , C22:3 13 , $\mathrm{C} 22: 5 \omega 3$ and $\mathrm{C} 22: 6 \omega 3)$ represented, after correction for paired initial value (time-zero) and even after $4 \mathrm{~h}$ of incubation, no more than $35.7 \%$ of the total amount of the same $\omega 3$ fatty acids initially present in the triglycerides, as distinct $(\mathrm{P}<0.005)$ at $69.7 \pm 3.8 \%(n=4)$ in the case of C16:0, C18:0, C18:1w9 and C20:1(09). A different situation prevailed, however, with the two other emulsions (MCT:FO and MLF), in which case the fractional release of the $\omega 3$ fatty acids (C20:5 $13, \mathrm{C} 22: 5 \omega 3$ and $\mathrm{C} 22: 6 \omega 3)$ at $240 \mathrm{~min}$ was much higher, amounting to $69.0 \pm 3.3 \%(n=2)$. In this respect, the difference between 
the FO emulsion, on one hand, and the MCT:FO and MLF emulsions, on the other hand, was obvious in the case of both $\mathrm{C} 20: 5 \omega 3$ and $\mathrm{C} 22: 6 \omega 3$. The fractional release of these fatty acids from the triglycerides was, at $240 \mathrm{~min}$, indeed twice higher $(201.2 \pm 13.5 \% ; \mathrm{n}=4$; $\mathrm{P}<0.005)$ with the MCT:FO and MLF emulsions than the corresponding value found for the same fatty acid with the FO emulsion.

A higher total amount of unesterified fatty acids (at the exclusion of $\mathrm{C} 8: 0$ and $\mathrm{C10:0)}$ accumulated in the medium during incubation of the MLF emulsion, as compared to the MCT:FO emulsion (Fig. 3, upper panels). This can be accounted for by the much higher initial amount of C16 and C18 fatty acids in the triglyceride fraction of the MF emulsion $(231.4 \mu \mathrm{g} / \mathrm{ml})$ as compared to the MCT:FO emulsion $(25.1 \mu \mathrm{g} /$ $\mathrm{ml}$ ). The generation of unesterified $\mathrm{C} 16$ and $\mathrm{C} 18$ fatty acids, relative to their initial concentration in the triglyceride fraction, was indeed quite efficient. This is documented in the lower panels of Fig. 3, using C16:0 as an illustrative example. The fractional release of $\mathrm{C} 16: 0$ was always higher than that of C22:6w3. Between 120 and $240 \mathrm{~min}$, the fractional release of $\mathrm{C} 22: 6 \omega 3$ from the triglycerides only represented $82.9 \pm 5.0 \%$ $(\mathrm{n}=6 ; \mathrm{P}<0.02)$ of the paired value found at the same time of incubation and with the same emulsion in the case of C16:0.

Essentially comparable results were obtained when the generation of unesterified fatty acids over $4 \mathrm{~h}$ of incubation was expressed relative to their paired initial concentration in both diglycerides and triglycerides. Thus, the fractional release of $\mathrm{C} 20: 5 \omega 3$ and $\mathrm{C} 22: 6 \omega 3$ now averaged, respectively, 22.4 \pm 1.0 and $63.6 \pm 2.8 \%$ ( $n=3$ in both cases) of the paired value found with the same emulsion in the case of C16:0. Moreover, relative to the corresponding fractional release of each of these three fatty acids found with the MCT:FO emulsion, that observed with the FO and MLF emulsions averaged, respectively, $50.5 \pm 3.7 \%$ and $102.0 \pm 3.0 \%$ ( $n=3$ in both cases). In considering the biochemical significance of these findings, it should be kept in mind that the initial total amount of the two $\omega 3$ fatty acids under consideration represented in the MCT:FO and MLF emulsions only $17.9 \pm 0.4$ and $9.8 \pm 0.7 \%$ ( $n=2$ in both cases) of that found in the FO emulsion. In the case of $\mathrm{C} 16: 0$, such an initial total amount ranged from $9.4 \mu \mathrm{g} /$ $\mathrm{ml}$ in the MCT:FO emulsion to $18.3 \mu \mathrm{g} / \mathrm{ml}$ in the FO emulsion and $30.9 \mu \mathrm{g} / \mathrm{ml}$ in the MLF emulsion.

When expressed relative to the total amount $(\mu \mathrm{g} / \mathrm{ml})$ of unesterified fatty acids (at the exclusion of C8:0 and C10:0) measured after 240-min incubation, the relative contribution of $\mathrm{C} 16: 0, \mathrm{C} 18: 0$ and $\mathrm{C} 18: 1 \omega 9$ represented, with the FO and MLF emulsion respectively, $1.98 \pm 0.15$ and $1.15 \pm 0.06$ times the initial relative contribution of the same fatty acids (again calculated at the exclusion of C8:0 and C10:0) in triglycerides ( $n=3$ in both cases). Thus, as judged by this criterion, the relative extent of deesterification of these three fatty acids represented, with the MLF emulsion, only $58.6 \pm 4.1 \%$ of the paired value found for the same fatty acid in the FO emulsion $(\mathrm{n}=3 ; \mathrm{P}<0.01$ versus unity). The corresponding values found for C16:0 and C18:1 19 in the MCT:FO emulsion were close to those recorded in the MLF emulsion, with a paired MCT:FO/ MLF ratio of $90.4 \pm 2.5 \%$ ( $n=2 ; P>0.1$ versus unity).

Phospholipids. The fate of phospholipids was also examined in this first set of experiments. In this respect, essentially comparable results ( $\mathrm{n}=3$ in all cases) were recorded with the three emulsions. Over $4 \mathrm{~h}$ of incubation, the total concentration of fatty acids associated with phospholipids slowly decreased, eventually reaching a value representing $80.5 \pm 1.6 \%$ of the paired initial concentration, i.e. $25.5 \pm 0.8 \mu \mathrm{g} / \mathrm{ml}$.

As shown in Table I, the major fatty acids initially present in the phospholipids were $\mathrm{C} 16: 0$ (relative abundance on a ponderal basis: $36.7 \pm 0.8 \%), \mathrm{C} 18: 0(23.4 \pm 0.8 \%), \mathrm{C} 18: 1 \omega 9$ $(17.2 \pm 0.9 \%), \mathrm{C} 18: 2 \omega 6(11.3 \pm 0.4 \%)$ and $\mathrm{C} 20: 4 \omega 6(7.6 \pm 1.4 \%)$. These relative abundances were little affected during incubation.

As judged from the absolute values $(\mu \mathrm{g} / \mathrm{ml})$ for the amount of fatty acids identified in the phospholipid fraction, a sizeable decrease in this concentration over $240 \mathrm{~min}$ of incubation was only observed in the case of $\mathrm{C} 16: 0$ and $\mathrm{C} 18: 0$, with a mean value for such a decrease of $2.67 \pm 0.30$ and $1.34 \pm 0.07 \mu \mathrm{g} / \mathrm{ml}$, respectively (Table II). Relative to the paired initial amount of these fatty acids in the phospholipids, this decrease corresponded to fractional hydrolysis of $28.5 \pm 2.6 \%$ and $22.5 \pm 1.1 \%$ in the case of $\mathrm{C} 16: 0$ and $\mathrm{C} 18: 0$. This suggests a somewhat lower lability of the ester bound involving C18:0, as compared to $\mathrm{C} 16: 0$, in these phospholipids. In the former case, the fractional hydrolysis indeed only represented $79.6 \pm 3.2 \%(\mathrm{P}<0.025)$ of the paired value found with $\mathrm{C} 16: 0$.

In order to assess the respective contribution of phospholipid and glyceride hydrolysis to the total generation of unesterified C16:0 and C18:0, the decrement of these fatty acid contents $(\mu \mathrm{g} / \mathrm{ml})$ in phospholipids, after 240-min incubation, was expressed as a percentage of the paired total amount of the same fatty acid lost from all lipid fractions (phospholipids, diglycerides, monoglycerides). As shown in Table II, such a percentage was quite variable, ranging between the extreme values of $11.2 \%$ (in the case of the generation of C16:0 from the MLF emulsion) and 100.0\% (in the case of the generation of C18:0 from the MCT:FO emulsion). This coincided with the fact that these two situations were also characterized by the extreme values for the initial content of glycerides (diglycerides and triglycerides) in the same fatty acids, i.e. $30.9 \mu \mathrm{g} / \mathrm{ml}$ for C16:0 in the MLF emulsion and 0 for the C18:0 initial content of glycerides in the MCT:FO emulsion. As a matter of fact, and as expected, there was a significant negative correlation $(\mathrm{r}=-0.8172 ; \mathrm{n}=6 ; \mathrm{P}<0.05)$ between the fractional contribution of phospholipid hydrolysis (logarithmic values), estimated as indicated above, and the initial amount of each fatty acid under consideration in the diglyceride and triglyceride fractions of each emulsion.

\section{Experiment 2}

Triglycerides. Fig. 4 (upper panel) illustrates the time course for the decrease in triglyceride concentration during incubation. As already observed in the first set of experiments, the initial concentration of triglycerides and the rate of lipolysis were both lower in the case of the FO emulsion than with the MCT:FO or MLF emulsions. Such a difference remained evident when considering separately the fate of distinct fatty acids. For instance, at $120 \mathrm{~min}$, when sizeable amounts of the concerned fatty acids could still be measured in the triglyceride fraction, the residual concentration $(\mu \mathrm{g} / \mathrm{ml})$ of C16:0, C20:5 13 and C22:6 13 , when expressed relative to the corresponding initial concentration (time-zero), represented, in 

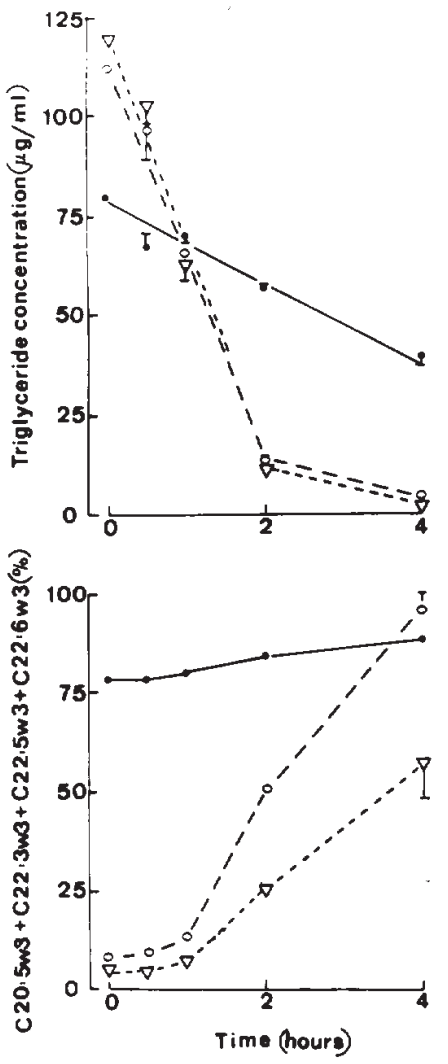

Figure 4. Time course for the changes in triglyceride concentration and composition in the FO (closed circles and solid line), MCT:FO (open circles and dashed line) and MLF (triangles and dotted line) emulsion (Expt. 2). Upper panel: the absolute concentration of triglycerides is ranged on a cartesian scale. Lower panel: relative contribution of $\mathrm{C} 20: 5 \omega 3, \mathrm{C} 22: 5 \omega 3$ and $\mathrm{C} 22: 6 \omega 3$ to the total amount of fatty acids in the triglyceride fraction. Mean values $( \pm$ SEM, whenever exceeding the size of the symbols for the mean value) refer to 2-3 individual measurements.

the case of the MCT:FO and MLF emulsions, only $67.8 \pm 9.5 \%$ $\mathrm{n}=17 ; \mathrm{P}<0.025)$ of that found, with the same fatty acid and at the same time, in the case of the FO emulsion.

Significant differences were also observed when comparing the fate of distinct fatty acids in the same emulsion(s). After only 60-min incubation, at a time when the concentration $(\mu \mathrm{g} / \mathrm{ml})$ of the long-chain polyunsaturated $\omega 3$ fatty acid (C20:5 13 and $\mathrm{C} 22: 6 \omega 3)$ in the triglyceride fraction was not yet significantly affected, the concentrations of $\mathrm{C} 8: 0$ and C10:0 were already markedly decreased to $67.2 \pm 2.0 \%$ and $36.5 \pm 2.0 \%$ ( $n=6$ in both cases) of the paired initial value in the MCT:FO and MLF emulsions, respectively. Relative to the initial concentration, that recorded at 60 min was slightly but significantly lower $(\mathrm{P}<0.05)$ in the case of $\mathrm{C} 8: 0$ than in that of $\mathrm{C} 10: 0$, the former value averaging $89.0 \pm 3.4 \%(n=6)$ of the mean corresponding value found with the same emulsion in the case of $\mathrm{C10}: 0(100.0 \pm 3.5 \%$; $=6)$. This coincided with a progressive decrease of the paired $\mathrm{C} 8: 0 / \mathrm{C} 10: 0$ ratio in the triglycerides of both the MCT:FO and MLF emulsions, from an initial value of $1.692 \pm 0.017(n=4)$ to $1.635 \pm 0.004(n=6)$ at $30 \mathrm{~min}$ and $1.496 \pm 0.034(\mathrm{n}=6)$ at $60 \mathrm{~min}$ (pooled results obtained with the MCT:FO and MLF emulsions). These three ratios were indeed significantly different from one another $(\mathrm{P}<0.005$ or less $)$, indicating a greater hydrolytic sensitivity of the ester bound in the case of C8:0 as compared to C10:0.
The higher resistance of long-chain polyunsaturated $\omega 3$ fatty acids, as distinct from other fatty acids, to lipase-catalyzed hydrolysis resulted in a progressive increase of the relative contribution of these $\omega 3$ acids in the triglyceride fraction during incubation (Fig. 4, lower panel). In the case of the FO emulsion, such an increase was attributable to a parallel rise in the relative contribution of $\mathrm{C} 20: 5 \omega 3$, whilst that of $\mathrm{C} 22: 3 \omega 3$ $(1.9 \pm 0.1 \%), \mathrm{C} 22: 5 \omega 3(5.0 \pm 0.1 \%)$ and $\mathrm{C} 22: 6 \omega 3(36.2 \pm 0.3 \%)$ remained virtually unchanged throughout incubation $(n=13$ in all cases). The increment in the relative contribution of all concerned $\omega 3$ fatty acids or only C20:5 $\omega 3$ above their respective initial values averaged, after $4 \mathrm{~h}$ of incubation, $9.5 \pm 0.6$ and $9.8 \pm 0.6 \%$ respectively ( $\mathrm{n}=3$ in both cases). This indicates that, in the FO emulsion, the ester bound of C20:5 03 was most resistant to hydrolysis. In the MCT:FO and MLF emulsions, the relative contribution of $\mathrm{C} 20: 5 \omega 3$ and $\mathrm{C} 22: 6 \omega 3$, which were the sole long-chain $\omega 3$ fatty acids identified in triglycerides, also displayed a progressive increase, at least up to $120 \mathrm{~min}$ of incubation. At each time point, the relative contribution of these $\omega 3$ fatty acids in the triglyceride fraction was approximately twice higher in the MCT:FO emulsion than in the MLF emulsion, in good agreement with the relative richness of fish oil in these two emulsions.

In all cases, the fractional hydrolysis of C22:6 103 was higher than that of $\mathrm{C} 20: 5 \omega 3$. For instance, at $120 \mathrm{~min}$, when sizeable amounts of these fatty acids could still be measured in the triglycerides of the three emulsions, the residual concentration $(\mu \mathrm{g} / \mathrm{ml})$, relative to the paired initial value, averaged in the case of $\mathrm{C} 22: 6 \omega 3$ only $70.4 \pm 9.9 \%(\mathrm{n}=9 ; \mathrm{P}<0.03)$ of the corresponding value found in the case of $\mathrm{C} 20: 5 \mathrm{\omega} 3$ $(100.0 \pm 7.0 \% ; \mathrm{n}=9$; pooled data obtained with the three emulsions). This coincided with a progressive increase during incubation of the paired $\mathrm{C} 20: 5 \omega 3 / \mathrm{C} 22: 6 \omega 3$ ratio above its initial value $(0.99 \pm 0.03 ; n=6)$ in all three emulsions. The magnitude of such an increase was inversely related to the concentration of fish oil in each emulsion. For instance, at $120 \mathrm{~min}$, the $\mathrm{C} 20: 5 \omega 3 / \mathrm{C} 22: 6 \omega 3$ ratio averaged $1.05 \pm 0.02$ in the FO emulsion, as distinct $(\mathrm{P}<0.05$ or less) from $1.15 \pm 0.02$ in the MCT:FO emulsion and 1.34 \pm 0.02 in the MLF emulsion $(n=2-3)$. At $240 \mathrm{~min}$ the same ratio averaged $1.25 \pm 0.03$ in the FO emulsion, as compared $(\mathrm{P}<0.02)$ to $1.61 \pm 0.08$ in the MCT:FO emulsion ( $n=3$ in both cases), whilst $C 22: 6 \omega 3$ was no longer detected in the triglycerides of the MLF emulsion.

Diglycerides. The initial amount of diglycerides was grossly proportional to the relative amount of fish oil in each emulsion, averaging $39.3 \pm 0.1 \mu \mathrm{g} / \mathrm{ml}$ in the FO emulsion, $4.7 \pm 0.3 \mu \mathrm{g} / \mathrm{ml}$ in the MCT:FO emulsion and $1.6 \pm 0.7 \mu \mathrm{g} / \mathrm{ml}$ in the MLF emulsion ( $\mathrm{n}=2$ in each case). As documented in Fig. 5 (upper panel), the concentration of diglycerides progressively decreased during incubation in the case of the FO emulsion. In the case of the MCT:FO and MLF emulsions, however, a transient increase in the concentration of diglycerides was observed before their eventual disappearance.

The fatty acid composition of the diglycerides was also vastly different when comparing the $\mathrm{FO}$ emulsion to either the MCT:FO or MLF emulsion (Fig. 5, lower panel). In the case of the FO emulsion, the diglyceride-associated fatty acids were mainly the $\omega 3$ fatty acids $(\mathrm{C} 20: 5 \omega 3, \mathrm{C} 22: 5 \omega 3$ and $\mathrm{C} 22: 6 \omega 3)$. They accounted for $78.3 \pm 1.5 \%(n=2)$ of the total 

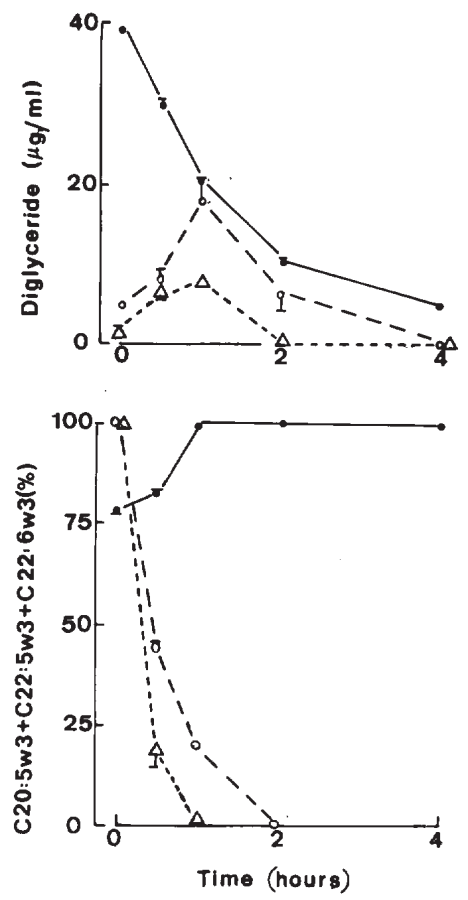

Figure 5. Time course for the changes in diglyceride concentration and composition in the FO (closed circles and solid line), MCT:FO (open circles and dashed line) and MLF (triangles and dotted line) emulsion (Expt. 2). Upper panel: the absolute concentration of diglycerides is ranged on a cartesian scale. Lower panel: relative contribution of $\mathrm{C} 20: 5 \omega 3, \mathrm{C} 22: 5 \omega 3$ and $\mathrm{C} 22: 6 \omega 3$ to the total amounts of fatty acids in the diglyceride fraction. Mean values $( \pm$ SEM, whenever exceeding the size of the symbol for the mean value) refer to 2-3 individual determinations.

amount of fatty acids at the onset of incubation, for $82.5 \pm 1.2 \%$ $(n=3)$ at $30 \mathrm{~min}$ and for $100 \%(n=9)$ from 60 min of incubation onwards. In the case of the MCT:FO and MLF emulsions, the relative contribution of $\mathrm{C} 20: 5 \omega 3$ and $\mathrm{C} 22: 6 \omega 3$, which were the sole identified $\omega 3$ fatty acids, amounted to $100 \%$ $(n=4)$ at the onset of incubation. It then rapidly decreased during incubation (Fig. 5, lower panel). This coincided with the appearance of sizeable amounts of C8:0 and C10:0 and, in a few samples, of $\mathrm{C} 16: 0$ in the diglycerides generated by the MCT:FO and MLF emulsions. The paired C20:5 $133 /$ $\mathrm{C} 22: 6 \omega 3$ ratio of the diglycerides present in the $\mathrm{FO}$ emulsion progressively increased during incubation from an initial value of $0.969 \pm 0.001(\mathrm{n}=2)$ to $1.009 \pm 0.013$ at $30 \mathrm{~min}, 1.104 \pm 0.007$ at $60 \mathrm{~min}, 1.382 \pm 0.025$ at $120 \mathrm{~min}$ and $1.705 \pm 0.013$ at $240 \mathrm{~min}$ ( $n=3$ in all cases), once again documenting the resistance towards hydrolysis under the present experimental conditions of the ester bound involving $\mathrm{C} 20: 5 \omega 3$. As long as they could be quantified in a reliable manner, the paired C20:5 $13 / \mathrm{C} 22: 6 \omega 3$ ratio of the diglycerides found in the other emulsions also progressively increased during incubation. For instance, in the case of the MCT:FO emulsion, such a paired ratio increased from an initial value of $1.022 \pm 0.010(\mathrm{n}=2)$ to $1.081 \pm 0.013$ at $30 \mathrm{~min}$ and $1.105 \pm 0.025$ at $60 \mathrm{~min}(\mathrm{n}=3$ in both cases) in fair agreement with the data obtained in the case of the FO emulsion.

Unexpectedly, the paired $\mathrm{C} 8: 0 / \mathrm{C} 10: 0$ ratios in the diglycerides found at 30 and $60 \mathrm{~min}$ in the MCT:FO and MLF emulsions were significantly higher than those initially found in the triglycerides of these emulsions. They indeed averaged, in the case of the MCT:FO and MLF emulsions, respectively, $2.126 \pm 0.039$ and $2.138 \pm 0.020$ ( $\mathrm{n}=6$ in both cases) as distinct from $(\mathrm{P}<0.005$ or less $)$ from $1.706 \pm 0.019$ and $1.678 \pm 0.032$ $(\mathrm{n}=2$ in both cases) for the initial $\mathrm{C} 8: 0 / \mathrm{C} 10: 0$ paired ratio in the corresponding triglycerides. In the case of the diglycerides generated from the MCT:FO emulsion, the C8:0/C10:0 paired ratio decreased, however, to $1.365 \pm 0.037(\mathrm{n}=3)$ at $120 \mathrm{~min}$, again suggesting a greater lability of the ester bound involving C8:0 as compared to C10:0.

The fractional release of fatty acids was higher from diglycerides than from triglycerides. For instance, with the FO emulsion and after $2 \mathrm{~h}$ of incubation, the residual concentration $(\mu \mathrm{g} / \mathrm{ml})$ of $\mathrm{C} 20: 5 \omega 3$ and $\mathrm{C} 22: 6 \omega 3$, expressed relative to the corresponding initial value (time-zero), averaged $36.8 \pm 3.1 \%$ in the diglyceride fraction, as distinct $(\mathrm{P}<0.001)$ from $76.0 \pm 1.2 \%$ in the triglyceride fractions ( $n=6$ in both cases). In the case of C16:0 and C18:0 and at the same time, such a residual concentration amounted to $69.6 \pm 1.7 \%(n=6)$ in the triglyceride fraction, whilst a sizeable amount of these two fatty acids was no longer detected in the diglyceride fraction.

Monoglycerides and phospholipids. In this second set of experiments, the monoglycerides and phospholipids were examined together without prior separation of these two classes of lipids. As documented in Table III their initial concentration, which was not significantly different with the three emulsions, averaged $8.14 \pm 0.39 \mu \mathrm{g} / \mathrm{ml} \quad(\mathrm{n}=6)$. It represented $\sim 30 \%$ of the concentration found in the first experiment for the same lipids, in fair agreement with the 4-fold difference in the total concentrations of glycerides in these two experiments. With all three emulsions, the concentration of monoglycerides and phospholipids progressively increased during incubation reaching its peak value after $120 \mathrm{~min}$ in the case of the MCT:FO and MLF emulsions; whilst still increasing between 120 and 240 min with the FO emulsion. This increase reflects most likely the accumulation of monoglycerides. With the FO emulsion it indeed coincides with a progressive decrease in the fractional contribution of C16:0, C18:0 and C18:1w9, the occasional appearance of minor amounts of $\mathrm{C} 18: 2 \omega 6, \mathrm{C} 20: 3 \omega 6, \mathrm{C} 20: 4 \omega 6$ and $\mathrm{C} 22: 5 \omega 3$, and a marked increase in the fractional contribution of C20:5 03 and C22:6 03 . The latter increase was more sustained and, in relative terms, more pronounced in the case of $\mathrm{C} 20: 5 \omega 3$ than in that of C22:6w3. Likewise, with both the MCT:FO and MLF emulsions, the relative contribution of C16:0, C18:0 and C18:1 $\omega 9$ progressively decreased during incubation, whilst that of $\mathrm{C} 20: 5 \omega 3$ and $\mathrm{C} 22: 6 \omega 3$ progressively increased above its initial 0 value. Moreover, with the latter two emulsions, sizeable amounts of C8:0 and C10:0 accumulated in the lipid classes under consideration. With the MLF emulsions, a progressive rise in both the absolute amounts or relative contribution of $\mathrm{C} 18: 2 \omega 6$ in the monoglycerides and phospholipid fraction was observed reaching its peak value after 120 min of incubation (Table II).

At this point, two findings merit particular attention. First, with all three emulsions, the $\mathrm{C} 20: 5 \omega 3 / \mathrm{C} 22: 6 \omega 3$ paired ratio in the phospholipid and monoglyceride fraction progressively increased as a function of the length of incubation. This suggests that the monoglycerides generated during incubation were first mainly esters of $\mathrm{C} 22: 6 \omega 3$, with a delayed and later 
Table III. Concentration of fatty acids associated with monoglycerides (MG) and phospholipids (PL) in the second set of experiments.

\begin{tabular}{|c|c|c|c|c|c|}
\hline Time (min) & 0 & 30 & 60 & 120 & 240 \\
\hline \multicolumn{6}{|l|}{ FO emulsion } \\
\hline $\mathrm{MG}+\mathrm{PL}(\mu \mathrm{g} / \mathrm{ml})$ & $8.59 \pm 0.91$ & $10.76 \pm 0.35$ & $15.94 \pm 0.18$ & $23.35 \pm 1.75$ & $27.84 \pm 0.73$ \\
\hline $\mathrm{C} 16: 0(\%)$ & $35.7 \pm 0.4$ & $28.9 \pm 0.8$ & $20.5 \pm 0.1$ & $14.3 \pm 0.9$ & $11.1 \pm 0.2$ \\
\hline C18:0 (\%) & $25.1 \pm 0.4$ & $20.6 \pm 0.4$ & $15.1 \pm 0.1$ & $10.7 \pm 0.7$ & $8.4 \pm 0.2$ \\
\hline 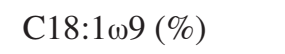 & $17.7 \pm 0.1$ & $15.2 \pm 0.2$ & $11.5 \pm 0.2$ & $8.2 \pm 0.6$ & $6.4 \pm 0.1$ \\
\hline $\mathrm{C} 18: 2 \omega 6(\%)$ & - & - & $2.1 \pm 2.1$ & - & - \\
\hline $\mathrm{C} 20: 3 \omega 6(\%)$ & - & $2.4 \pm 2.4$ & $3.2 \pm 1.6$ & $1.1 \pm 1.1$ & $3.0 \pm 0.2$ \\
\hline 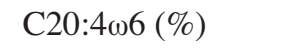 & - & - & $1.6 \pm 1.6$ & $2.9 \pm 1.5$ & $4.5 \pm 0.1$ \\
\hline $\mathrm{C} 20: 5 \omega 3(\%)$ & $8.0 \pm 1.1$ & $14.1 \pm 0.7$ & $21.5 \pm 0.2$ & $32.9 \pm 1.5$ & $42.0 \pm 1.0$ \\
\hline $\mathrm{C} 22: 5 \omega 3(\%)$ & - & - & $3.5 \pm 1.8$ & $2.5 \pm 1.3$ & $2.0 \pm 1.0$ \\
\hline $\mathrm{C} 22: 6 \omega 3(\%)$ & $13.5 \pm 0.4$ & $18.8 \pm 0.5$ & $21.1 \pm 0.4$ & $27.6 \pm 1.3$ & $22.5 \pm 0.4$ \\
\hline \multicolumn{6}{|l|}{ MCT:FO emulsion } \\
\hline $\mathrm{MG}+\mathrm{PL}(\mu \mathrm{g} / \mathrm{ml})$ & $7.98 \pm 0.95$ & $7.44 \pm 0.04$ & $12.50 \pm 0.98$ & $31.84 \pm 0.88$ & $29.56 \pm 0.19$ \\
\hline $\mathrm{C} 8: 0(\%)$ & - & - & $18.6 \pm 0.4$ & $36.4 \pm 0.0$ & $26.1 \pm 0.1$ \\
\hline C10:0 (\%) & - & - & $18.7 \pm 0.2$ & $31.1 \pm 0.2$ & $34.1 \pm 0.2$ \\
\hline $\mathrm{C} 16: 0(\%)$ & $45.5 \pm 0.8$ & $45.2 \pm 0.7$ & $27.0 \pm 1.5$ & $9.6 \pm 0.4$ & $10.4 \pm 0.1$ \\
\hline C18:0 (\%) & $31.2 \pm 1.5$ & $33.2 \pm 0.6$ & $19.4 \pm 1.0$ & $6.8 \pm 0.3$ & $7.6 \pm 0.1$ \\
\hline 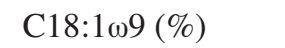 & $23.2 \pm 0.6$ & $21.6 \pm 0.9$ & $13.6 \pm 0.6$ & $5.2 \pm 0.2$ & $5.9 \pm 0.1$ \\
\hline $\mathrm{C} 20: 3 \omega 6(\%)$ & - & - & - & $0.7 \pm 0.7$ & - \\
\hline $\mathrm{C} 20: 5 \omega 3(\%)$ & - & - & - & $4.9 \pm 0.1$ & $8.9 \pm 0.0$ \\
\hline $\mathrm{C} 22: 6 \omega 3(\%)$ & - & - & $2.8 \pm 2.8$ & $5.2 \pm 0.1$ & $7.1 \pm 0.0$ \\
\hline \multicolumn{6}{|l|}{ MLF emulsion } \\
\hline $\mathrm{MG}+\mathrm{PL}(\mu \mathrm{g} / \mathrm{ml})$ & $7.83 \pm 0.4$ & $12.00 \pm 1.34$ & $20.06 \pm 1.76$ & $32.70 \pm 2.07$ & $27.39 \pm 0.38$ \\
\hline $\mathrm{C} 8: 0(\%)$ & - & $6.7 \pm 1.8$ & $23.6 \pm 1.9$ & $21.7 \pm 0.2$ & $13.2 \pm 0.1$ \\
\hline C10:0 (\%) & - & $5.6 \pm 0.8$ & $17.9 \pm 0.4$ & $20.8 \pm 0.2$ & $23.0 \pm 0.1$ \\
\hline C16:0 (\%) & $46.3 \pm 0.3$ & $29.7 \pm 1.2$ & $16.3 \pm 1.1$ & $8.7 \pm 0.2$ & $10.9 \pm 0.0$ \\
\hline C18:0 (\%) & $30.7 \pm 0.3$ & $19.5 \pm 1.7$ & $10.9 \pm 0.5$ & $6.0 \pm 0.2$ & $7.5 \pm 0.1$ \\
\hline 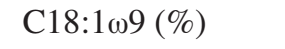 & $23.1 \pm 0.5$ & $16.6 \pm 1.4$ & $11.7 \pm 0.5$ & $10.2 \pm 0.2$ & $10.8 \pm 0.2$ \\
\hline $\mathrm{C} 18: 2 \omega 6(\%)$ & - & $11.3 \pm 1.0$ & $14.3 \pm 0.4$ & $22.7 \pm 0.2$ & $20.1 \pm 0.3$ \\
\hline $\mathrm{C} 18: 3 \omega 3(\%)$ & - & - & - & $0.9 \pm 0.9$ & - \\
\hline $\mathrm{C} 20: 2 \omega 6(\%)$ & - & $0.9 \pm 0.9$ & - & - & - \\
\hline $\mathrm{C} 20: 3 \omega 6(\%)$ & - & $1.2 \pm 1.2$ & - & $1.4 \pm 0.7$ & $2.9 \pm 0.1$ \\
\hline $\mathrm{C} 20: 4 \omega 6(\%)$ & - & $3.7 \pm 1.0$ & - & - & - \\
\hline $\mathrm{C} 20: 5 \omega 3(\%)$ & - & - & - & $3.2 \pm 0.2$ & $5.1 \pm 0.0$ \\
\hline $\mathrm{C} 24: 0(\%)$ & - & $1.6 \pm 1.6$ & - & - & - \\
\hline $\mathrm{C} 22: 5 \omega 3(\%)$ & - & $1.2 \pm 1.2$ & - & - & - \\
\hline $\mathrm{C} 22: 6 \omega 3(\%)$ & - & $2.1 \pm 2.1$ & $5.3 \pm 0.1$ & $4.2 \pm 0.2$ & $5.8 \pm 0.5$ \\
\hline
\end{tabular}

net accumulation of C20:5 13 monoglycerides. Second, in the case of the MCT:FO and MLF emulsions, respectively, the paired $\mathrm{C} 8: 0 / \mathrm{C} 10: 0$ ratio in the same fraction averaged at 240 min $0.767 \pm 0.005$ and $0.575 \pm 0.007$ ( $n=3$ in both cases), as distinct $(\mathrm{P}<0.001)$ from $1.085 \pm 0.040(\mathrm{n}=6)$ and $1.173 \pm 0.060$ $(n=9)$ at earlier sampling times. This finding is compatible with a more rapid generation of C8:0 monoglycerides as compared to C10:0 monoglycerides.

It should be stressed that in these experiments, the absolute concentrations of C16:0, C18:0 and C18:109 in the phospho- lipid and monoglyceride fractions usually remained fairly stable throughout the incubation and displayed comparable values with the three emulsions, suggesting that they corresponded mainly to the presence of these fatty acids in phospholipids. The two sole obvious exceptions to the stability of the absolute concentrations of these fatty acids in the phospholipid and monoglyceride fractions consisted, with the FO emulsion, in a modest increase $(\mathrm{P}<0.02)$ in the absolute amount of $\mathrm{C} 18: 1 \omega 9$ from a basal value of $1.67 \pm 0.05 \mu \mathrm{g} / \mathrm{ml}(\mathrm{n}=4)$ to a peak value $(120 \mathrm{~min})$ of $1.89 \pm 0.01 \mu \mathrm{g} / \mathrm{ml}(\mathrm{n}=3)$ and, with the MLF 
emulsion, in a more pronounced increase in the absolute amount of C18:1w9 in the same phospholipid and monoglyceride fractions from a comparable $(\mathrm{P}>0.35)$ basal value of $1.76 \pm 0.08 \mu \mathrm{g} / \mathrm{ml}(\mathrm{n}=2)$ to $1.96 \pm 0.05 \mu \mathrm{g} / \mathrm{ml}$ at $30 \mathrm{~min}$, $2.34 \pm 0.12 \mu \mathrm{g} / \mathrm{ml}$ at $60 \mathrm{~min}, 3.34 \pm 0.18 \mu \mathrm{g} / \mathrm{ml}$ at $120 \mathrm{~min}$ and $2.95 \pm 0.02 \mu \mathrm{g} / \mathrm{ml}$ at $240 \mathrm{~min}(\mathrm{n}=3$ in all cases). In these two instances, the increase in the absolute amount of C18:1 109 associated with phospholipids and monoglycerides displayed a comparable time course. When considering the results obtained with the three emulsions, there was an obvious parallelism between the magnitude of the changes in the absolute amounts of $\mathrm{C} 18: 1 \omega 9$ associated with the lipid classes under consideration and the relative initial content of the same fatty acid in the triglycerides (Table I). This strongly suggests that such changes corresponded to the time-related accumulation of monoglycerides derived from the triglycerides present in each emulsion. If one ignores the just-mentioned higher than basal values, the overall mean absolute amounts of C16:0, C18:0 and C18:1 199 in the phospholipid and monoglyceride fraction averaged, respectively, 3.21 $\pm 0.04 \mu \mathrm{g} / \mathrm{ml}(\mathrm{n}=40)$, $2.28 \pm 0.03 \mu \mathrm{g} / \mathrm{ml}(\mathrm{n}=40)$ and $1.70 \pm 0.02 \mu \mathrm{g} / \mathrm{ml}(\mathrm{n}=22)$.

Unesterified fatty acids. The time course for the total generation of unesterified fatty acids from the FO emulsion displayed an exponential pattern (Fig. 6, upper panel). Thus, each doubling of the incubation length resulted in an approximate 3-fold $(2.72 \pm 0.26$; d.f. $=10 ; \mathrm{P}<0.025$ versus 2.00$)$ increase in their concentration in the incubation media. The time-dependent pattern for the accumulation of unesterified fatty acids was not identical, however, in all cases. For instance, the paired ratio between $\mathrm{C} 20: 5 \omega 3$ and $\mathrm{C} 16: 0$ concentrations progressively increased from $0.99 \pm 0.03$ at $30 \mathrm{~min}$ to $1.31 \pm 0.01$ at $60 \mathrm{~min}$, $1.94 \pm 0.04$ at $120 \mathrm{~min}$ and $2.98 \pm 0.07$ at $240 \mathrm{~min}(\mathrm{n}=2-3)$.

The total production of unesterified fatty acids generated from the MCT:FO and MLF emulsions could not be reliably assessed because of the volatility of C8:0 and C10:0. The partial loss by evaporation of these medium-chain fatty acids was documented by the fact that their concentration in the incubation medium after $4 \mathrm{~h}$ of incubation represented, at the most, $55.4 \pm 2.4 \%(n=3)$ of the paired value for their disappearance from the triglyceride fraction. The latter percentage, which refers to the fate of $\mathrm{C} 10: 0$ generated from the MCT: FO emulsion, was higher $(\mathrm{P}<0.01)$ than that found in the case of C10:0 generated from the MLF emulsion $(43.0 \pm 0.3 \%$; $\mathrm{n}=3$ ); coinciding with a twice higher initial amount of this fatty acid in the triglycerides of the MCT:FO emulsion, as compared to the MLF emulsion (Table I). Likewise, in the case of C8:0, the recovery of the unesterified fatty acid in the medium, after $240 \mathrm{~min}$ of incubation, relative to the corresponding value for its disappearance from the triglyceride fraction was higher $(\mathrm{P}<0.025)$ with the MCT:FO emulsion $(30.5 \pm 2.3 \% ; n=3)$ than with the MLF emulsion (14.5 $\pm 3.8 \%$; $\mathrm{n}=3$ ). These percentages also document that, relative to their disappearance from the triglyceride fraction, the eventual recovery of these two fatty acids as unesterified molecules was significantly lower $(\mathrm{P}<0.025)$ in the case of $\mathrm{C} 8: 0$ as compared to C10:0, whether with the MCT:FO or MLF emulsion, as expected from the greater volatility of the former than latter fatty acid. For the purpose of comparison, in the case of C16:0, $\mathrm{C} 20: 5 \omega 3$ and $\mathrm{C} 22: 6 \omega 3$, the total amount $(\mu \mathrm{g} / \mathrm{ml})$ recovered at

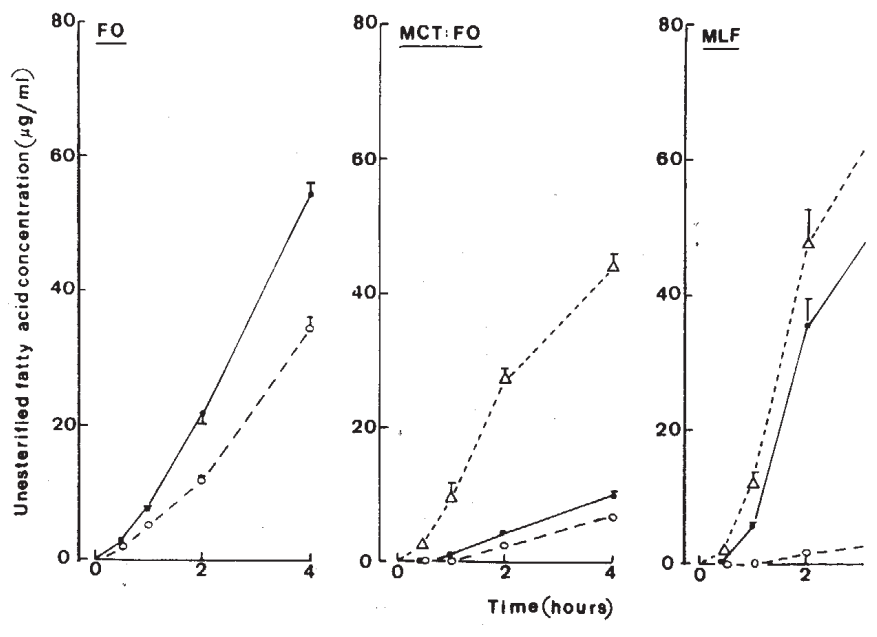

Figure 6. Time course for the changes in unesterified fatty acid concentration in the FO (left), MCT:FO (middle) and MLF (right) emulsions (Expt. 2). The total concentration of fatty acids refers to the measurements made inclusive (triangles and dotted line) or not (closed circles and solid line) of $\mathrm{C} 8: 0$ and $\mathrm{C} 10: 0$. The concentration of $\mathrm{C} 20: 5 \omega 3, \mathrm{C} 22: 5 \omega 3$ and $\mathrm{C} 22: 6 \omega 3$ is indicated by the open circles and dashed line. Mean values ( \pm SEM, whenever exceeding the size of the symbol for the mean value) refer to 2-3 individual determinations.

60,120 and 240 min in triglycerides, diglycerides, monoglycerides, phospholipids and unesterified fatty acids, when expressed relative to the mean corresponding value recorded in the first two samples ( 0 and $30 \mathrm{~min})$, averaged ( $\mathrm{n}=9$ in all cases) $100.7 \pm 1.2 \%$ in the FO emulsion, $101.9 \pm 2.8 \%$ in the MCT:FO emulsion and $98.0 \pm 2.3 \%$ in the MLF emulsion, yielding an overall mean value of $100.2 \pm 1.3 \%(n=27)$.

The partial loss of the medium-chain fatty acids, especially C8:0, also resulted in the case of the MCT:FO and MLF emulsions, in an apparently greater fractional release of C10:0 than $\mathrm{C} 8: 0$ ( $\mathrm{P}<0.005$ in both cases). Indeed, as judged from the residual amounts $(\mu \mathrm{g} / \mathrm{ml})$ of unesterified C8:0 and C10:0 measured in the medium after $4 \mathrm{~h}$ of incubation and the corresponding initial content (also expressed as $\mu \mathrm{g} / \mathrm{ml}$ ) of triglycerides in the same fatty acids, their apparent fractional release averaged, with the MCT:FO and MLF emulsions (pooled data), $20.2 \pm 3.0 \%$ in the case of $\mathrm{C} 8: 0$ as compared $(\mathrm{P}<0.001)$ to $46.0 \pm 1.0 \%$ in the case of $\mathrm{C} 10: 0$ ( $\mathrm{n}=6$ in both cases). This artefactual situation obviously conflicts with the evidence obtained from the measurements of triglycerideassociated C8:0 and C10:0, which unambiguously document the greater lability of the ester bound involving C8:0 as compared to C10:0.

Nevertheless, the computation of the total amount of unesterified fatty acids generated from each emulsion takes into account the measurement of unesterified C8:0 and C10:0 (Fig. 6), in order both to provide some quantitative information, even if underestimated, on the total production of free fatty acids and to depict grossly its time course.

As already mentioned for the FO emulsion, the time course for the generation of unesterified fatty acids (including C8:0 and C10:0) from the MCT:FO and MLF emulsions displayed an exponential pattern, at least up to 120 min (Fig. 6, upper right panels). Thus, each doubling of the incubation length resulted, during the first $2 \mathrm{~h}$ of incubation, in a 4 -fold 


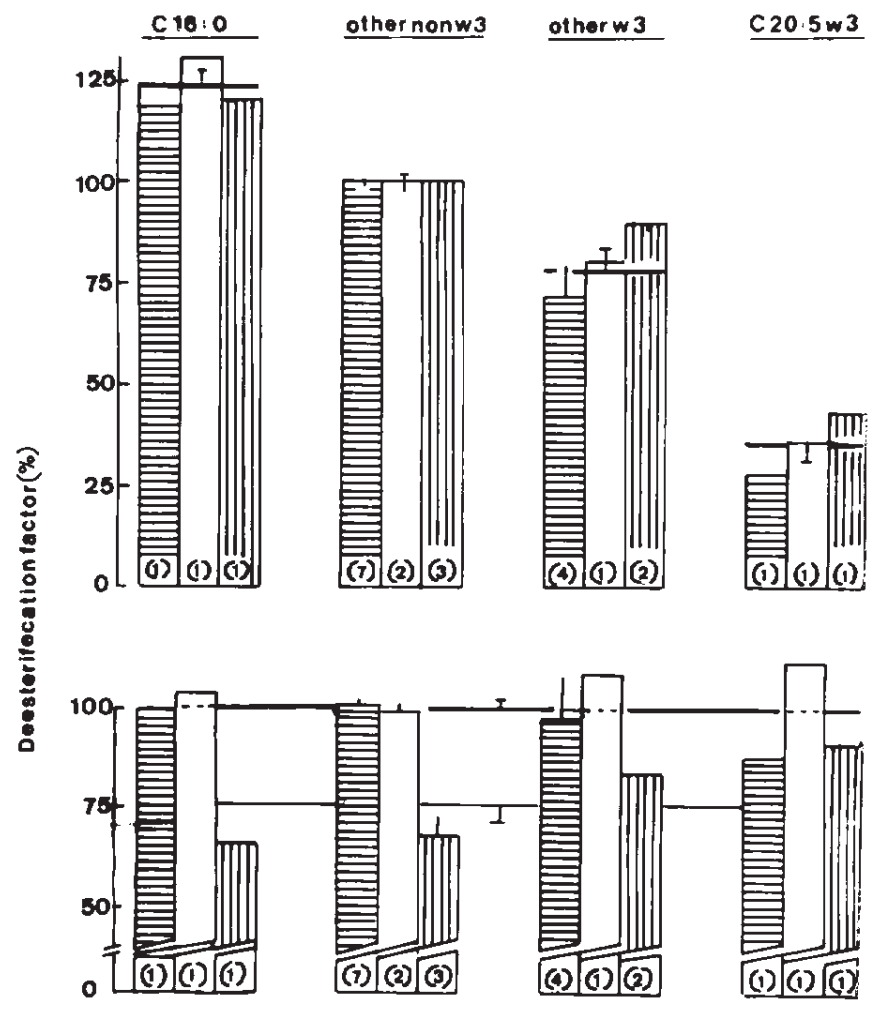

Figure 7. Deesterification of distinct fatty acids from triglycerides in the FO (horizontally-hatched columns), MCT:FO (open columns) and MLF (vertically-hatched columns) emulsion (Expt. 2). The deesterification factor represents the ratio between the relative contribution of each fatty acid to the total amount of unesterified fatty acids (at the exclusion of C8:0 and C10:0) at $240 \mathrm{~min}$ and the relative contribution of the same fatty acid to the total amount of fatty acids (at the exclusion of C8:0 and C10:0) in the triglyceride fraction at time-zero. It is expressed relative to either the mean value found, with the same emulsion, for all non- $\omega 3$ fatty acids other than C16:0 (upper panel) or the mean value found, with the same fatty acid(s), in the FO and MCT:FO emulsions (lower panel). Mean values ( \pm SEM) refer to the number of fatty acid(s) under consideration in each case, as indicated in parentheses at the bottom of each column. The heavy solid lines refer to the overall mean values ( \pm SEM indicated by the $\mathrm{T}$ bars) found for each individual fatty acid (C16:0 and $\mathrm{C} 20: 5 \omega 3)$ or each group of fatty acids (other non- $\omega 3$ and other $\omega 3$ fatty acids) either with the three emulsions (upper panel) or with the FO and MCT:FO emulsions (upper heavy line in the lower panel) as compared to the MLF emulsion (lower heavy line in the lower panel). In the upper panel, the $100 \%$ reference value corresponds to a mean deesterification factor of $1.564 \pm 0.035(\mathrm{n}=7)$ in the FO emulsion, $1.541 \pm 0.049(\mathrm{n}=2)$ in the MCT:FO emulsion, and $1.072 \pm 0.066(\mathrm{n}=3)$ in the MLF emulsion.

$(4.18 \pm 0.50 ; \mathrm{n}=12 ; \mathrm{P}<0.001)$ increase in the total concentration of unesterified fatty acids. However, over the last $2 \mathrm{~h}$ of incubation, the increase in the total concentration of unesterified fatty acids, expressed as the paired 240/120 $\mathrm{min}$ ratio, decreased $(\mathrm{P}<0.001)$ to only $1.60 \pm 0.11(\mathrm{n}=6)$, a value significantly lower $(\mathrm{P}<0.02)$ than the theoretical value of 2.00 , itself based on the assumption of a constant production rate of unesterified fatty acids. A comparable pattern was observed when the data relative to $\mathrm{C} 8: 0$ and $\mathrm{C} 10: 0$ were no longer considered. In this case, the concentrations of unesterified fatty acids generated from the MCT:FO and MLF emulsions were $7.09 \pm 1.49$ times $(n=5)$ higher at 120 min than $60 \mathrm{~min}$, but only underwent a 2-fold increase (multiplication factor: 2.01 \pm 0.20 ; $\mathrm{n}=6$ ) between 120 and $240 \mathrm{~min}$, the difference between these two multiplication factors being highly significant $(\mathrm{P}<0.001)$.
With the MCT:FO and MLF emulsions, like with the FO emulsion, the time course for the generation of distinct fatty acids was quite variable. For instance, the paired ratio between $\mathrm{C} 20: 5 \omega 3$ and $\mathrm{C} 16: 0$ concentrations progressively increased during incubation from 0 at 30 and $60 \mathrm{~min}$ to $0.59 \pm 0.05$ (120 $\mathrm{min})$ and $1.34 \pm 0.05$ (240 $\mathrm{min})$ with the MCT:FO emulsion and to $0.03 \pm 0.00(120 \mathrm{~min})$ and $0.14 \pm 0.00(240 \mathrm{~min})$ with the MLF emulsion. When comparing these values to those already mentioned in the case of the FO emulsion, it became obvious that the value for such a paired ratio reached after $4 \mathrm{~h}$ of incubation was tightly related to $\mathrm{C} 20: 5 \omega 3 / \mathrm{C} 16: 0$ ratio in the corresponding triglycerides. For instance, there was a 20fold difference for the $\mathrm{C} 20: 5 \omega 3 / \mathrm{C} 16: 0$ paired ratio in both the fatty acids released in the incubation medium (0.14 versus $2.98)$ and those initially present in the triglycerides $(0.38$ versus 8.01 ), when considering the MLF versus the FO emulsions. After $4 \mathrm{~h}$ of incubation, the concentration of unesterified long-chain $\omega 3$ fatty acids in the incubation medium was, with the three emulsions under consideration, virtually identical, when expressed by reference to their relative initial abundance in the triglycerides.

In order to assess the relative extent of deesterification of distinct fatty acids, the mean contribution ( $n=3$ in all cases) of each fatty acid (expressed as a ponderal percentage) to the overall generation of unesterified fatty acids (at the exclusion of C8:0 and C10:0) recorded after 240-min incubation was divided by the mean corresponding contribution of the same fatty acid (also expressed as a ponderal percentage) in the triglyceride fraction at the onset of incubation $(n=2$ in all cases). As illustrated in the upper panel of Fig. 7, the quotient of such a division, here referred to as a deesterification factor, was always higher $(\mathrm{P}<0.05$ or less) with $\mathrm{C} 16: 0$ than for all other fatty acids. Pooling all available data, it averaged $123.3 \pm 3.7 \%(n=3 ; P<0.001)$ of the mean corresponding value found with the same emulsion for all other non- $\omega 3$ fatty acids $(100.0 \pm 1.9 \% ; n=12)$. Relative to the latter reference value, the deesterification factor averaged $78.1 \pm 5.2 \%(n=7)$ with the $\omega 3$ fatty acids (at the exclusion of $\mathrm{C} 20: 5 \omega 3$ ) and $35.8 \pm 4.3 \%$ $(\mathrm{n}=3)$ with $\mathrm{C} 20: 5 \omega 3$. The difference between these mean percentages was always highly significant $(\mathrm{P}<0.005$ or less).

It should be underlined, however, that the deesterification factors found with the MLF emulsion were almost always lower than the corresponding factors found with either the FO or MCT:FO emulsions (Fig. 7, lower panel). They averaged $76.2 \pm 4.2 \%(n=7 ; P<0.001)$ of the mean values found for the same type or same class of fatty acid(s) with the latter two emulsions $(100.0 \pm 2.7 \%$; $n=17)$. Such a difference was most marked in the case of the non- $\omega 3$ fatty acids, with a mean value of $68.3 \pm 3.0 \%(n=4)$ for the MLF emulsion as compared $(\mathrm{P}<0.001)$ to a reference value of $100.0 \pm 1.6 \%(\mathrm{n}=11)$ derived from 8 measurements made with the $\mathrm{FO}$ emulsion and 3 measurements made with the MCT:FO emulsion. Relative to a comparable reference value $(100.0 \pm 6.3 \% ; n=7)$, the deesterification factors found with the $\omega 3$ fatty acids of the MLF emulsion averaged $86.7 \pm 2.8 \%(n=3)$, the difference between these two sets of data failing to achieve statistical significance. Nevertheless, when the same analysis considered all individual measurements (rather than a single mean value for each fatty acid), a significant difference $(\mathrm{P}<0.02$ or less) was found between the results obtained with the FO emulsion 
and/or MCT:FO emulsion and those computed for the MF emulsion, at least in the case of $\omega 3$ fatty acids other than C20:5 03 .

It could be argued that, in the case of the FO emulsion, the estimation of the fractional release of fatty acids should be based on the data relative to the fate of both triglycerides and diglycerides. However, when such a calculation was performed, essentially comparable results were obtained. As a matter of fact, the difference between the fate of C16:0 and $\mathrm{C} 20: 5 \omega 3$, relative to that of all other non- $\omega 3$ fatty acids, was even somewhat more pronounced than that illustrated in Fig. 7.

\section{Discussion}

The present results document that, under the present experimental conditions, all three emulsions under consideration were suitable for the generation of unesterified polyunsaturated

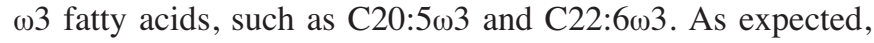
however, the absolute amount of these fatty acids generated during incubation was mainly ruled by the respective abundance of these $\omega 3$ fatty acids in each emulsion. For instance, as judged from their disappearance from the triglyceride fraction after 240-min incubation in the two sets of experiments, the generation of these $\omega 3$ fatty acids $(\mu \mathrm{g} / \mathrm{ml})$ represented, with the MCT:FO and MLF emulsion respectively, only $24.8 \pm 2.9 \%$ and $15.5 \pm 2.0 \%$ ( $n=4$ in both cases) of the paired value found with the FO emulsion. Nevertheless, the latter two percentages were higher $(\mathrm{P}<0.05)$ than the corresponding percentages for the absolute amounts of the same $\omega 3$ fatty acids in the triglyceride fraction at the onset of incubation, i.e. $16.3 \pm 0.4 \%$ and $10.2 \pm 0.3 \%$ ( $n=4$ in both cases) in the case of the MCT:FO and MLF emulsion, respectively, relative to the value found in the FO emulsion (100.0\%). The paired ratio between the percentages relative to the generation of each fatty acid and their initial content in the triglyceride fraction was indeed in all cases higher with the MCT:FO and MLF emulsion than with the FO emulsion. For instance, in the case of the MCT: FO emulsion, such a paired ratio averaged, relative to the corresponding value found with the FO emulsion, 1.766 \pm 0.039 and $1.254 \pm 0.043$ in the first and second set of experiments $(n=2$ in both cases). Pooling all available data relative to the

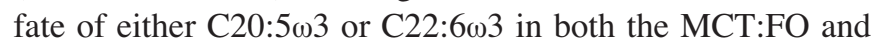
MLF emulsion, this paired ratio averaged, relative to the corresponding value found within the same experiment with the FO emulsion, $1.515 \pm 0.108$ ( $\mathrm{n}=8 ; \mathrm{P}<0.005$ versus unity). Thus, the generation of these two $\omega 3$ fatty acids relative to their concentration in the initial triglyceride fraction was, as judged from the latter mean ratio, $\sim 50 \%$ higher in the MCT: FO and MLF emulsions than in the FO emulsion. A comparable situation prevailed in the case of diglycerides. Indeed, the fractional release of $\mathrm{C} 20: 5 \omega 3$ and $\mathrm{C} 22: 6 \omega 3$ from the diglycerides initially present in the MCT:FO and MLF emulsion represented, at $240 \mathrm{~min}$, at least $124.2 \pm 2.9 \%(\mathrm{n}=8$; $\mathrm{P}<0.001$ relative to unity) of the corresponding value found within the same experiment with the FO emulsion. The latter percentage is underestimated since, in 6 out of 8 cases, either the concerned fatty acid or all diglycerides had disappeared at 240 min from the incubation medium containing the MCT: FO or MLF emulsion.
The fractional release of fatty acids, relative to their initial concentration in each lipid fraction, was higher in diglycerides than in triglycerides. This was documented in the two sets of experiments and in the case of both non- $\omega 3$ (C16:0 and 18:0) and $\omega 3$ (C20:5 $\omega 3$ and $\mathrm{C} 22: 6 \omega 3)$ fatty acids. The phospholipids present in the emulsions were most resistant to lipoprotein lipase-catalyzed hydrolysis.

The fractional release of fatty acids from each lipid fraction (triglycerides, diglycerides, monoglycerides and even phospholipids) differed vastly with distinct fatty acids. The following hierarchy was found in terms of the lability of the ester bound: C8:0 > C10:0 > C16:0 > other non- $\omega 3$ fatty acids > $\mathrm{C} 22: 6 \omega 3$ and other long-chain polyunsaturated $\omega 3$ fatty acids (at the exclusion of C20:5 13 ) > C20:5 23 .

The following two series of observations were rather unexpected. First, after 30-60 min of incubation, the paired C8:0/C10:0 ratio in diglycerides was significantly higher than that found at $0 \mathrm{~min}$ or later in the triglyceride fraction. Such a ratio usually reached its peak value in the diglyceride fraction after 60-min incubation and then progressively decreased to eventually reach values well below those initially found in the triglyceride fraction. This late fall probably reflects the higher lability of the ester bound involving C8:0, as compared to C10:0. However, the factor(s) responsible for the high C8:0/C10:0 ratio found after 30-60 min of incubation in the diglycerides generated from the MCT:FO or MLF emulsion remains a matter of speculation. It could reflect heterogeneity among the medium-chain triglycerides in these two emulsions, with the presence of a limited amount of triglycerides in which the position of C8:0 and/or C10:0 and, possibly, the identity and/or position of other fatty acids would favour the generation of diglycerides with a higher relative content in C10:0, relative to that of $\mathrm{C} 8: 0$, as that initially found in the bulk of triglycerides. This interpretation is supported by the fact that, as a rule, the paired $\mathrm{C} 8: 0 / \mathrm{C} 10: 0$ ratio progressively increased in the diglyceride fraction over the first $60 \mathrm{~min}$ of incubation.

Second, an unexpected difference between the two sets of experiments consisted in the fact that the deesterification index, especially that of non- $\omega 3$ fatty acids, was lower in the MLF emulsion than in the MCT:FO emulsion in the second set of experiments (paired MLF/MCT:FO ratio for C16:0 and C18:1 $\omega 9$ averaging $65.2 \pm 1.1 \%$; $n=2$ ), whilst such was not the case $(\mathrm{P}<0.01)$ in the first set of experiments (paired MLF/MCT:

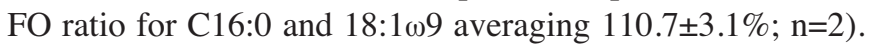
These values are derived from the relative contribution of the two fatty acids, relative to the total amount of fatty acids (at the exclusion of C8:0 and C10:0) in both the unesterified fatty acids (at $240 \mathrm{~min}$ ) and triglycerides (at $0 \mathrm{~min}$ ). Yet, the absolute concentrations $(\mu \mathrm{g} / \mathrm{ml})$ of unesterified fatty acids,

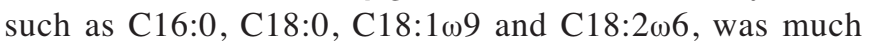
higher in the first than in the second set of experiments. For instance, the total absolute amount of the four fatty acids under consideration that accumulated in the incubation medium after $4 \mathrm{~h}$ of incubation was $5.49 \pm 1.47(\mathrm{n}=2)$ times higher in the first set of experiments than in the second (mean value derived from the data collected with either the MCT:FO or MLF emulsion). However, the MLF/MCT:FO ratio for the total amount of these four fatty acids generated over $4 \mathrm{~h}$ of incubation was almost twice higher in the second set of 
experiments (MLF/MCT:FO ratio of 14.01) than in the first set of experiments (MLF/MCT:FO ratio of 8.03). Since these fatty acids may cause inhibition of lipoprotein lipase catalytic activity, the latter difference could conceivably account for the lower deesterification index found with the MLF emulsion, as compared to the MCT:FO emulsion, in the second, but not first, set of experiments. It should be kept in mind, however, that the two sets of experiments differed from one another in other respects, e.g. by the human plasma sample used to supply apoproteins to the lipid emulsions.

In conclusion, the present study affords novel information on the effect of lipoprotein lipase upon the fate of fatty acids in three distinct lipid emulsions. The results obtained with the MCT:FO emulsion are obviously relevant to the use of this emulsion in human subjects, as outlined in the introduction.

\section{Acknowledgements}

This study was supported by a grant from the Région de Bruxelles-Capitale (BC-BR 246/2410) to Target Hit s.a. (Brussels, Belgium). The lipid emulsions were provided by Braun Melsungen (Melsungen, Germany). We are grateful to A. Chwalik and A. Dufour for technical assistance and C. Demesmaeker for secretarial help.

\section{References}

1. Portois L, Deckelbaum RJ, Malaisse WJ and Carpentier YA: Accumulation rapide d'eicosapentaénoate dans les phospholipides cellulaires après injection intraveineuse d'une émulsion d'huile de poisson et de triglycérides à chaîne moyenne à des sujets normaux. Nutr Clin Metab 18 (suppl 1): S53, 2004.

2. Simoens C, Richelle M, Rössle C, Derluyn M, Deckelbaum RJ and Carpentier YA: Manipulation of tissue fatty acid profile by intravenous lipids in dogs. Clin Nutr 14: 177-188, 1995. 